\title{
ESTRATEGIAS DE DESARROLLO ECONÓMICO LOCAL PARA EL MUNICIPIO DE GIRARDOTA
}

\author{
Local economic development strategies for the Municipality of Girardota
}

Iván Dario Rojas-Arenas

MSc en Gestión de la Innovación Tecnológica, Cooperación y Desarrollo Regional, Institución Universitaria

Pascual Bravo, Medellín-Colombia, ivan.rojasar@pascualbravo.edu.co

\section{John Alejandro Cardona-Quintero}

PhD en Desarrollo local y economía social, Instituto para el Desarrollo de Antioquia, Medellín-Colombia, johncardona24@yahoo.com.ar

\section{José Benjamín Gallego-Alzate}

MSc en Ciencias Económicas, Instituto Tecnológico Metropolitano, Medellín-Colombia, josegallego@itm.edu.co

\section{Cómo citar / How to cite}

Rojas-Arenas, I. D., Cardona-Quintero, J.A. y Gallego-Alzate, J.B. (2017). Estrategias de desarrollo económico local para el municipio de Girardota. Revista CEA, 3(6), 29-45.

Recibido: 6 de febrero de 2017

Aceptado: 15 de mayo de 2017

\section{Resumen}

El desarrollo económico endógeno supone un cambio de paradigma en la forma de pensar el desarrollo en las regiones, en un intento por explicar los procesos de acumulación y generación de riqueza, y el papel que juegan los actores locales en estos procesos. En el presente artículo, proponemos unas estrategias de desarrollo económico local para el Municipio de Girardota (del Departamento de Antioquia- Colombia), obtenidas a partir de una investigación participativa que incluyó un diagnóstico, con el fin de identificar las vocaciones económicas, y así definir objetivos y estrategias que puedan ser ejecutadas a partir de programas y proyectos. Las estrategias formuladas apuntaron a la solución de cuatro problemas fundamentales de la localidad: desempleo, baja calidad y poca pertinencia del sistema educativo, competitividad menguada en sectores claves de la economía local y escasos niveles de participación ciudadana. Las conclusiones a las que llegamos muestran la importancia de la participación real de la comunidad en la elaboración de estrategias de desarrollo alineadas con los intereses y necesidades de los distintos actores locales.

Palabras clave: desarrollo económico endógeno, actores locales, participación ciudadana, estrategias de desarrollo local.

\begin{abstract}
Endogenous economic growth is a paradigm shift in the conception of regional development in an attempt to explain wealth accumulation and creation and the role of local stakeholders in these processes. This article proposes local economic development strategies for the Municipality of Girardota (Department of Antioquia, Colombia) derived from participatory research that included an analysis
\end{abstract}


to identify economic vocations, objectives and strategies that can be implemented with programs and projects. The proposed strategies aim to solve four fundamental problems in the district: unemployment, poor quality and pertinence of the education system, low competitiveness of key sectors of the local economy, and scarce citizen participation. Our conclusions reveal the importance of actual participation of the community in the creation of development strategies that are in line with the interests and needs of different local stakeholders.

Keywords: endogenous economic growth, local stakeholders, citizen participation, local development strategies.

\section{INTRODUCCIÓN}

Durante las últimas décadas ha tomado importancia el estudio de los procesos de desarrollo económico de los territorios que evidencian que las regiones han alcanzado un nivel de desarrollo, aprovechando los recursos endógenos y la capacidad de actores locales para liderar dichos procesos. Actualmente, se habla del desarrollo económico local y el desarrollo económico endógeno como la manera en que las comunidades organizadas en algunos países han afrontado los retos que suponen la globalización de la economía. De acuerdo con esto, el objetivo de este artículo es presentar los resultados de la investigación participativa tendiente a proponer estrategias de desarrollo económico local para el municipio de Girardota en el Departamento de Antioquia (Colombia), se utiliza la metodología planteada por Ivan Silva Lira (2003), la cual ha sido ampliamente utilizada en la gestión estratégica del desarrollo local y regional por parte del ILPES; esta metodología se fundamenta en el análisis estratégico y la formulación de estrategias construidas en forma participativa con los actores empoderados que apunten a la solución de problemáticas en sus respectivas regiones.

Dentro del componente teórico, se hace una contextualización del desarrollo económico local, entendido este como una manera de explicar los procesos de transformación económica de algunas regiones a partir del aprovechamiento de sus recursos locales, lo que está muy relacionado con la noción de desarrollo económico endógeno y la participación de la comunidad, como lo mencionan varios autores (Alburquerque, 2004; Barroso y Flores, 2010, Dini, 2010), lo que presupone un proceso de cambio socioproductivo del territorio "desde abajo», desde los actores locales, quienes se encargan de auto liderar el proceso de crecimiento mediante la potenciación del recurso humano disponible. La metodología empleada constó de las siguientes fases en campo: diagnóstico, identificación de vocaciones, formulación de objetivos, elaboración de estrategias de desarrollo local y bosquejo del plan de inversión. Complementariamente, a través de talleres realizados con distintos actores locales, se construyó la matriz de importancia y gobernabilidad (Godet, 1993), con estos resultados se procedió a la construcción de la matriz de potencialidades, limitaciones y problemas para establecer las vocaciones y participativamente determinar los objetivos estratégicos y específicos, haciendo uso del árbol de problemas y de medios-fines para finalmente definir las estrategias de desarrollo local.

\section{MARCO TEÓRICO}

\section{Desarrollo Económico Local (DEL)}

Dentro del llamado paradigma funcionalista, se entendía el territorio como un espacio geográfico donde ocurrían eventos que daban lugar al desarrollo económico, pero pasaba por 
alto la interpretación de aspectos propios de la estructura social de cada región y no explicaba, de forma satisfactoria, los procesos de acumulación de capital al no evidenciar de forma clara su dinámica económica (Vázquez, 2007, pp. 183-210). En este sentido, el territorio era considerado como un delimitador de hechos económicos, pero no se hablaba de este como un factor clave en el proceso de desarrollo, entendiéndolo como el lugar en donde se establecen las relaciones entre los distintos actores desde la perspectiva de agentes económicos. Este proceso relacional entre actores, recursos y territorio es lo que da lugar al desarrollo económico local DEL, un concepto que surge al explicar cómo distintas comunidades y regiones buscaron la manera de satisfacer sus necesidades a partir de iniciativas de uso de los recursos locales, en donde las personas e instituciones hacen parte activa del proceso de transformación económica (Quispe y Ayaviri, 2013). En este sentido, se entiende el territorio como un recurso estratégico, el cual puede definir las oportunidades socioeconómicas de una localidad, al ser el lugar de una dinámica territorial determinada por sus instituciones y el conjunto de recursos potenciales con los que dispone (Brunet y Baltar, 2010).

Dentro del análisis del desarrollo local se deben tener en cuenta aspectos como la identidad territorial, dado que cuando una comunidad se identifica fuertemente con su sistema de creencias y costumbres, y los factores que determinan las características propias de la localidad, se favorece la formación de un entorno para el desarrollo, además de la cultura técnica, definida como «la elaboración, transmisión y acumulación de prácticas, conocimientos, normas y valores relacionados con una actividad económica, que contribuye a la regulación social en el sistema productivo local» (Romero, 2003, p. 82). Bigham y Myer
(1993) definen el DEL como una manera de aumentar la riqueza a través de la generación de empleo y la utilización óptima de los recursos que dispone una región. Furio Blasco (2004) interpreta el DEL como una salida a la crisis económica, presente en un mundo global y bajo una economía de mercado inclemente con algunos territorios, haciendo énfasis en las Pyme, el empresarismo y la gestión de la innovación. Alburquerque (2004), por su parte, habla de la manera en que se establecen las relaciones entre los actores locales y cómo estas propician la generación del DEL como proceso de cambio estructural que supone la movilización de los recursos locales aunada a la participación ciudadana y del surgimiento de relaciones entre agentes que interactúan para propiciar estrategias y acciones para la generación de ventajas competitivas dinámicas, teniendo como marco de actuación un objetivo de desarrollo local formulado conjuntamente. De allí que los procesos de desarrollo sean únicos para cada lugar (Vázquez, 2009), ya que no solo tienen en cuenta la dotación de recursos naturales y físicos, sino también la dinámica de las interacciones entre agentes y la manera como las sociedades condicionan sus procesos de desarrollo a partir de sus particularidades. Alburquerque (2004) plantea que el DEL se da en aquellas sociedades que presentan las condiciones para su surgimiento y que depende de factores como la cultura, aspectos sociales y códigos de conducta. El mismo autor (Alburquerque, 2004) habla de la aplicación de factores intangibles como las relaciones laborales, estilos de dirección, cooperación entre empresas, recurso humano, redes entre actores locales y cultura emprendedora. En definitiva, se pueden resumir las dimensiones del DEL en: económica, recursos humanos, socio-cultural, político-administrativa y ambiental (Pérez, 2008; Romero, 2003; Tello, 2006). 


\section{Desarrollo Económico Endógeno y DEL}

Cuando se menciona el desarrollo endógeno, se hace énfasis en la capacidad que tiene una comunidad de aprovechar los recursos internos (externos complementariamente) para su desarrollo. Lo que es una coincidencia con el DEL, frente a esto, Alburquerque (2004), opina que la estrategia de desarrollo local debe tener la capacidad de endogeneizar los recursos externos, vinculándolos de forma eficiente dentro de la dinámica del mercado interno y de acumulación de capital. Es así como el carácter de endógeno se funde con el proceso de desarrollo local obedeciendo a una lógica "desde abajo», donde, al igual que en el DEL, los actores locales juegan un papel determinante en el momento de definir los objetivos de desarrollo que apuntan a un mejoramiento estructural, convirtiéndose así, en una estrategia que implica la formulación y ejecución de programas y proyectos orientados al aprovechamiento de los recursos de la región (Quispe y Ayaviri, 2013), así como de los exógenos. Aunque por su extensión este tema se debe tratar en otro artículo.

Vázquez-Barquero (2007) complementa la noción, expresando que la comunidad local es la que se encarga de liderar y potenciar el proceso de crecimiento y cambio estructural, a través del aprovechamiento de los recursos del territorio, la generación de valor agregado, la introducción de innovaciones y la transformación del sistema productivo tradicional en uno competitivo.

Patricio Vergara (2004) analiza los temas faltantes para la construcción de un cuerpo teórico -sin terminar aún, valga la aclaraciónque implique una definición del desarrollo económico endógeno; distintos autores han aportado a este esfuerzo (Alburquerque, 2008; Boissier, 1997; Furio-Blasco, 1994; Quispe y Ayaviri, 2013; Vázquez-Barquero, 2007; Vergara, 2004), coincidiendo en algunos aspectos que hacen parte de su conceptualización. En primer lugar, se trata de un proceso de cambio estructural, innovador y emprendedor, en el cual se da importancia a los mecanismos de acumulación de capital y crecimiento en función de las características del territorio, lo cual explica que se presenten distintos caminos hacia el desarrollo de cada región; lo que, a la larga, implica una distinción con respecto a otras teorías económicas.

La motivación principal del proceso DEL es mejorar el nivel de vida a través del aprovechamiento de los recursos endógenos y estudios posteriores han adicionado, la capacidad para atraer y aprovechar recursos exógenos que complementen los primeros en el tiempo, respetando la autonomía de la comunidad, eso sí, dejando de lado la dependencia de las políticas asistenciales.

El segundo elemento de la conceptualización trata sobre la necesidad de generar economías de escala creando y potenciando redes de cooperación, incentivando la formación de clusters para el desarrollo; se podría hacer énfasis en que lo importante no es la cantidad de recursos disponibles en un territorio, sino la capacidad de las personas para hacer uso eficiente de los mismos como respuesta creativa a los retos que implica el modelo económico mundial desde los territorios, lo cual ubica a la sociedad civil participativa como el eje de transformación de la economía local. Entre otros factores del desarrollo endógeno se tienen: las instituciones, las iniciativas locales, la planificación pública y privada, las políticas de desarrollo, los procesos y procedimientos, la cultura y valores, el espíritu de trabajo, la capacidad de ahorro, los grupos sociales, las normas y mecanismos de regulación, la gestión de las innovaciones y el conocimiento, la flexibilidad del sistema productivo y la capacidad de las instituciones locales para afrontar el cambio. 
Frente al concepto de desarrollo económico endógeno se han suscitado algunas críticas por parte de distintos autores (Añez, 2006; Gorenstein y Burachik, 1999; Romero, 2003; Vázquez, 2009), como son: las barreras a los sistemas productivos territoriales y la dificultad para estos compitan de forma exitosa en un sistema económico globalizado; la capacidad que pueda tener el desarrollo endógeno para convertirse en una solución real para los problemas estructurales en países menos desarrollados; la dificultad para la creación de políticas claras en torno a los objetivos del desarrollo y las herramientas para lograrlo; la dificultad para coordinar los distintos actores locales por ejemplo el generar sinergias y cooperación entre pequeñas y medianas empresas; la falta de articulación entre las distintas políticas de desarrollo (local, regional, nacional); la poca autonomía de los entes locales dada la internacionalización de la economía; la falta de recursos humanos calificados para la transformación de los sistemas productivos. No obstante estas críticas, tener validez es más importante enfrentar el reto de ir mejorando la adaptabilidad y competitividad de los territorios a la nueva realidad económica mundializada.

\section{METOdOLOGÍA}

Esta investigación involucró a la población del municipio de Girardota, para que participara activamente en la ejecución en diferentes momentos; para este fin se utilizó la estrategia de talleres con los diferentes actores locales, a partir de estos se obtuvo información primaria, adicional, durante la construcción del plan de gobierno 2016-2019, se llevó a cabo una encuesta, en distintas zonas del municipio de Girardota, que tenía como objetivo preguntar a los habitantes de esta localidad y a representantes de distintas empresas del sobre los problemas que visualizaban y las posibles soluciones.

Las principales fuentes de información secundaria fueron: diagnósticos realizados en el plan de desarrollo 2016-2019, trabajos de investigación sobre desempeño fiscal y competitividad regional, caracterización de unidades productivas del municipio de Girardota, estudios de la Red de Empleo para el Área Metropolitana y el Plan Estratégico de Empleo para el Valle de Aburrá 2016-2028, así como datos estadísticos suministrados por el DANE. Con la información recolectada se reprodujo la propuesta de Iván Silva Lira (2003) sobre elaboración de estrategias de desarrollo económico local, la cual consta de 5 etapas: realización de diagnóstico, identificación de vocaciones, formulación de objetivos, elaboración de estrategias de desarrollo local, y propuestas de proyectos de inversión; a modo de complemento, con la información primaria se construyeron varias matrices analíticas.

\section{Matriz de Importancia vs Gobernabilidad - IGO}

Esta matriz se construyó a partir de la información obtenida en los talleres realizados con los actores locales, en donde se identificaban problemáticas relativas al municipio, se ponderaban y se planteaban posibles alternativas de solución. Dichas problemáticas se llevan a la gráfica de Importancia vs. Gobernabilidad, la cual establece cuatro cuadrantes, producto de la intersección entre la calificación media de importancia y gobernabilidad. En el primer cuadrante se ubican cuestiones estratégicas, muy importantes y gobernables; en el segundo, los retos, problemáticas muy importantes y poco gobernables; en el tercero y cuarto cuadrantes, problemáticas poco importantes.

Para la construcción de esta matriz se llevaron a cabo cinco talleres con distintos actores 
locales; se utilizó el modelo de matriz de importancia y dominio propuesto por Michel Godet (1993). En cada taller se realizaron las siguientes actividades: identificación de problemáticas del municipio, depuración y listado de dichas problemáticas, ponderación según importancia y gobernabilidad, y establecimiento de una zona de priorización de problemas en la matriz IGO, haciendo uso de una hoja de cálculo. Por último, se enunciaron los posibles proyectos que servirían para solucionar los problemas priorizados (retos y estratégicos). Ver anexo Tabla 8.

\section{Aplicación local de las herramientas de análisis}

Al inicio de la investigación en el territorio se recolectó la información a través de los talleres y las encuestas a los actores, posteriormente se construyó la Matriz de Importancia vs Gobernabilidad. A continuación, se muestran las gráficas de ponderación de problemas y Matriz IGO obtenidas en uno de los talleres (Figura 1, Tabla1). De igual manera, se anexa la Tabla 2, la cual contiene algunos indicadores de planificación integral, elaborados por el Área Metropolitana del Valle de Aburra. En las tablas 3 a la 7, se resumen los resultados de la implementación de la metodología propuesta pata este trabajo. En la Tabla 8 (Anexo) se hace un resumen de los talleres realizados con la comunidad.

\section{Diagnóstico}

La Tabla 3 muestra la Matriz de Potencialidades, Limitaciones y Problemas, organizada según áreas de interés y construida a partir del análisis de la información obtenida a partir de las distintas fuentes antes mencionadas.

\section{Vocaciones}

Las vocaciones obtenidas del análisis de la matriz de potencialidades, limitaciones $y$ problemas son: sector comercial y servicios, sector agrícola, sector industrial.

\section{Definición de los objetivos estratégicos y específicos}

Se definieron objetivos alcanzables, enfocados a sectores claves de la economía y que intenten establecer relaciones entre el mercado local, nacional e internacional; además, se analizaron los posibles inconvenientes que se presenten a futuro. Asimismo, desde la parte metodológica, se propone hacer énfasis en objetivos para el fomento del empleo local, la mejora de la calidad de vida de las personas, la diversificación de la economía y la mejora de infraestructuras (Silva, 2003). Para la formulación de objetivos, se usaron como herramientas los análisis de árboles de problemas, y de medios y fines. Los problemas a abordar son los siguientes: alto desempleo, baja calidad de la educación, poca competitividad de sectores claves de la economía local, poca participación ciudadana.

Se muestra el árbol de problemas para el tema de la poca participación ciudadana (Figura 2): Posteriormente se formularon los objetivos asociados a cada problema, se determinaron los resultados esperados y las actividades necesarias para alcanzar dichos resultados (Tabla 4). 
IGO

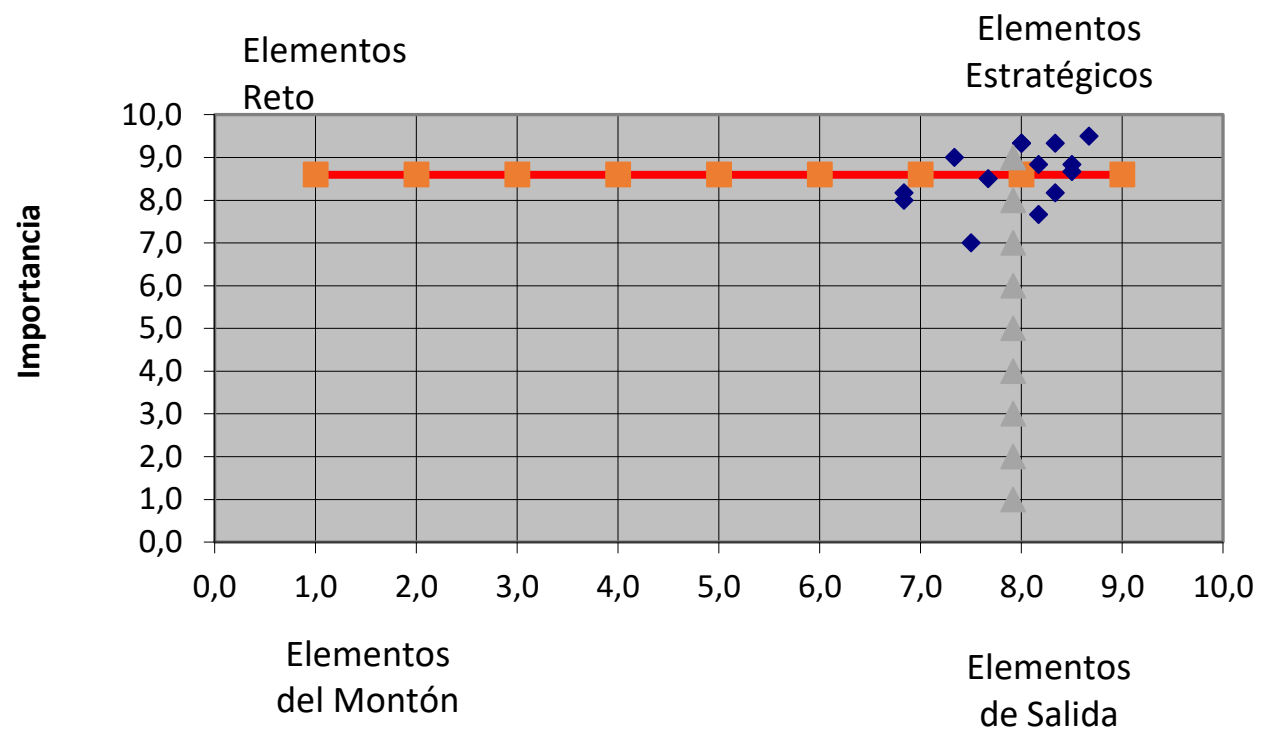

Gobernabiliad

Figura 1. Matriz IGO

Figure 1. Matrix IGO

Fuente: Alcaldía de Girardota, 2016.

Tabla 1. Ponderación Importancia vs Gobernabilidad

Table 1. Weighting Importance vs. Governance

\begin{tabular}{lcc}
\hline Problema & Importancia & Gobernabilidad \\
\hline Movilidad & 9.4 & 9.2 \\
\hline Contaminación & 9.0 & 9.1 \\
\hline Salud & 9.4 & 7.3 \\
\hline Desempleo & 9.3 & 9.3 \\
\hline Agua potable en veredas & 9.6 & 9.7 \\
\hline Déficit vivienda & 9.8 & 9.8 \\
\hline Violencia intrafamiliar & 9.6 & 9.1 \\
\hline Discriminación población LGTBI & 7.0 & 7.6 \\
\hline Baja calidad educativa & 9.3 & 8.1 \\
\hline Manejo finanzas publicas & 7.8 & 7.9 \\
\hline Deterioro social & 7.8 & 8.6 \\
\hline
\end{tabular}

Fuente: Alcaldía de Girardota, 2016. 
Tabla 2. Indicadores de Planificación Integral Table 2. Integral Planning Indicators

\begin{tabular}{|c|c|}
\hline \multicolumn{2}{|c|}{ Indicadores de Planificación Integral, Municipio de Girardota } \\
\hline Indicador/año medición & Resultado \\
\hline $\begin{array}{l}\text { Población Total (DANE) y Densidad Poblacional ( \# personas por } \\
\left.\mathrm{km}^{2}\right) / 2017\end{array}$ & 56,$755 ; 672.1$ \\
\hline Tasa neta de escolaridad superior (Por 100 personas)/2015 & 3.6 \\
\hline $\begin{array}{l}\text { Tasa neta de escolaridad educación básica secundaria y media (Por } \\
100 \text { personas)/2015 }\end{array}$ & 45.4 \\
\hline Tasa neta de escolaridad básica primaria (Por 100 personas)/2015 & 70.6 \\
\hline $\begin{array}{l}\text { Promedio de años de educación para personas entre } 15 \text { y más } \\
\text { años/2015 }\end{array}$ & 9.8 \\
\hline Tasa Bruta de Mortalidad/2016 & 3.7 \\
\hline Déficit cuantitativo de vivienda (Unidades)/2013 & $\begin{array}{l}105 \text { área urbana; } 151 \text { área } \\
\text { urbana }\end{array}$ \\
\hline $\begin{array}{l}\text { Porcentaje de hogares con necesidades básicas insatisfechas } \\
(\mathrm{NBI}) / 2015\end{array}$ & 4.4 área rural; 2.1 área urbana \\
\hline Porcentaje de hogares con alta dependencia económica/2015 & 1.1 área rural; 1.7 área urbana \\
\hline Índice de Condiciones de Vida/2013 & 68.7 área rural; 73.3 área urbana \\
\hline
\end{tabular}

Fuente: Área Metropolitana, 2017.

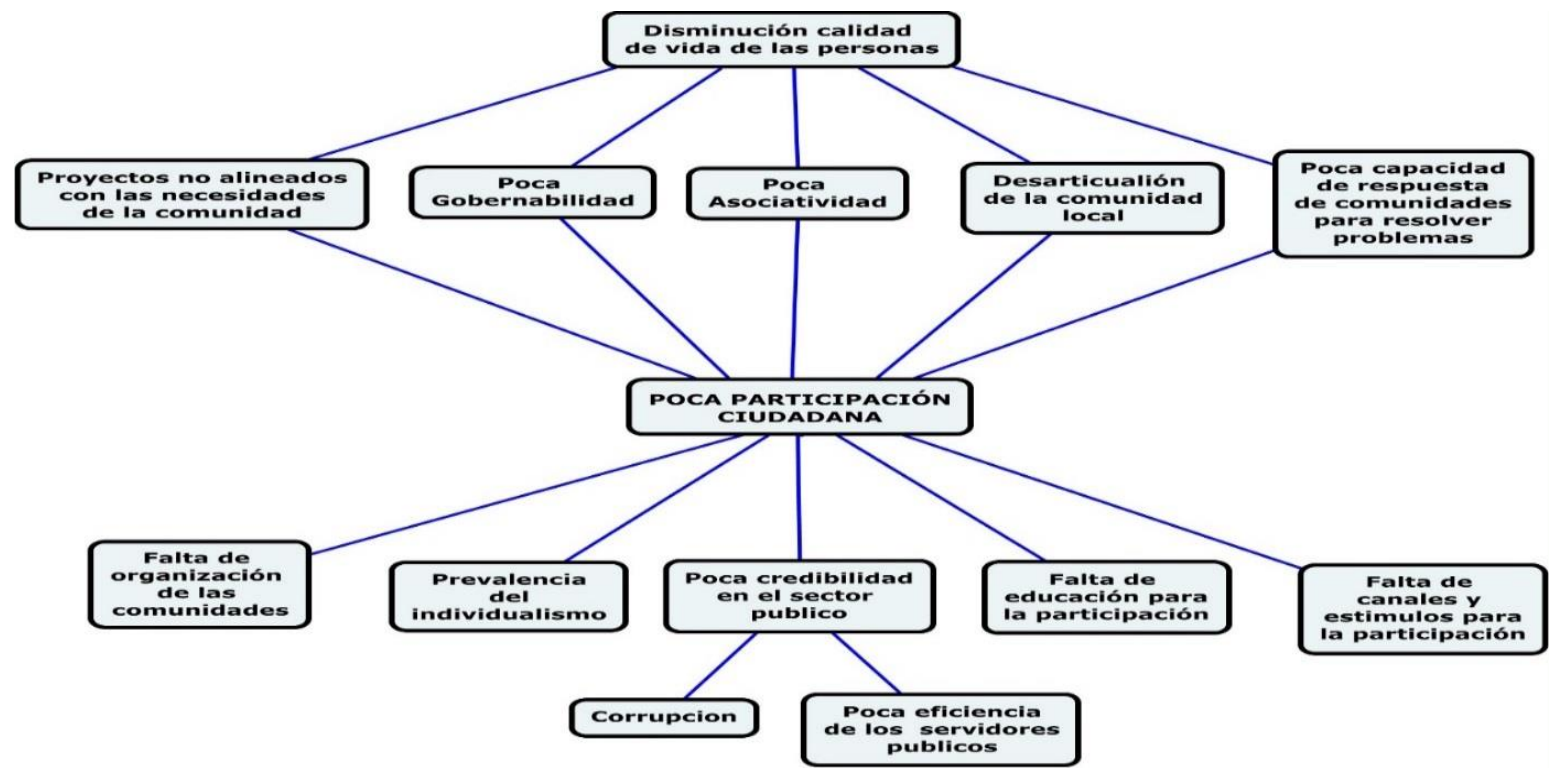

Figura 2. Árbol de problemas

Figure 2. Problem Tree

Fuente: elaboración propia. 
Tabla 3. Matriz de Potencialidades, Limitaciones y Problemas

Table 3. Matrix of Potentialities, Limitations and Problems

\begin{tabular}{|c|c|c|c|}
\hline Área temática & Potencialidades & Limitaciones & Problemas \\
\hline $\begin{array}{l}\text { Área } \\
\text { económica }\end{array}$ & 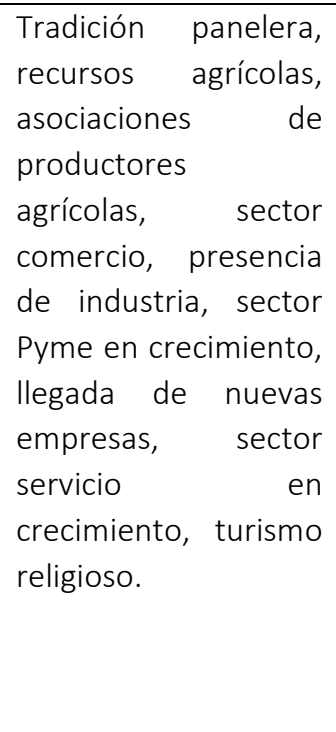 & $\begin{array}{l}\text { PEM no alineado con las } \\
\text { necesidades del sistema } \\
\text { productivo, cultura de } \\
\text { subsidiariedad } \\
\text { asistencialismo, bajos precios } \\
\text { agrícolas, poca preparación } \\
\text { académica de pequeños } \\
\text { empresarios, poca cultura de } \\
\text { emprendimiento, } \\
\text { informalidad en micro y } \\
\text { pequeñas empresas, difícil } \\
\text { acceso a financiamiento para } \\
\text { Pyme. }\end{array}$ & $\begin{array}{l}\text { Baja cultura asociativa, } \\
\text { cadenas de comercialización de } \\
\text { productos agrícolas, bajo nivel } \\
\text { de ingresos, tecnologías } \\
\text { obsoletas, poca } \\
\text { competitividad, poca } \\
\text { generación de valor agregado, } \\
\text { ausencia de procesos de } \\
\text { innovación einvestigación en la } \\
\text { industria local, poco trabajo } \\
\text { colaborativo entre empresas, } \\
\text { poca articulación del sistema } \\
\text { productivo local con la } \\
\text { administración, ausencia de } \\
\text { sistemas de apoyo a la } \\
\text { producción. }\end{array}$ \\
\hline $\begin{array}{ll}\text { Sistema físico } \\
\text { natural }\end{array}$ & $\begin{array}{l}\text { Ubicación estratégica } \\
\text { para el tránsito } \\
\text { comercial, turístico y } \\
\text { productivo, clima. }\end{array}$ & $\begin{array}{l}\text { Riesgos climáticos en invierno, } \\
\text { ausencia de un sistema } \\
\text { eficiente de gestión del riesgo. }\end{array}$ & Vías rurales en mal estado. \\
\hline $\begin{array}{l}\text { Demografía y } \\
\text { mercado de } \\
\text { trabajo }\end{array}$ & $\begin{array}{l}\text { PEA joven, programas } \\
\text { de emprendimiento y } \\
\text { empresarismo, } \\
\text { oficina del empleo, } \\
\text { unidades productivas } \\
\text { con procesos de } \\
\text { formación. }\end{array}$ & $\begin{array}{l}\text { Pocas oportunidades de } \\
\text { empleo, pocos recursos para } \\
\text { la promoción de programas } \\
\text { como «Capital Semilla», bajo } \\
\text { nivel de formación de } \\
\text { emprendedores. }\end{array}$ & $\begin{array}{l}\text { Aumento empleo informal, } \\
\text { desempleo, poco acceso de la } \\
\text { mujer al mercado laboral, altas } \\
\text { tasas de cesantía juvenil, mano } \\
\text { de obra poco calificada. }\end{array}$ \\
\hline Infraestructura & $\begin{array}{lr}\text { Parque } & \text { Educativo } \\
\text { Innova, } & \text { unidad } \\
\text { deportiva, } & \text { conexión } \\
\text { vial, parque industrial. }\end{array}$ & $\begin{array}{l}\text { Nivel de ingresos para } \\
\text { inversión en obras de } \\
\text { infraestructura. }\end{array}$ & $\begin{array}{l}\text { Falte de centros de acopio y } \\
\text { beneficiaderos para productos } \\
\text { agrícolas, nivel insuficiente de } \\
\text { estructuras para el desarrollo. }\end{array}$ \\
\hline $\begin{array}{l}\text { Aspectos } \\
\text { institucionales }\end{array}$ & $\begin{array}{l}\text { Política pública de } \\
\text { cultura, planes de } \\
\text { desarrollo, marcada } \\
\text { tradición religiosa. }\end{array}$ & $\begin{array}{l}\text { Poco apoyo para el fomento } \\
\text { de la cultura, poca cobertura } \\
\text { en salud y saneamiento } \\
\text { básico, bajo desempeño fiscal. }\end{array}$ & $\begin{array}{l}\text { Nula inversión en investigación, } \\
\text { deterioro ambiental, poca } \\
\text { participación de la comunidad, } \\
\text { baja calidad de la educación, } \\
\text { PEM y PEI y desarticulados con } \\
\text { el sector productivo, ausencia } \\
\text { de IES local. }\end{array}$ \\
\hline
\end{tabular}

Fuente: elaboración propia. 
Tabla 4. Objetivos

Table 4. Objectives

\begin{tabular}{|c|c|c|c|}
\hline \multicolumn{4}{|c|}{ Objetivo general: Mejorar la calidad de vida de los habitantes de Girardota } \\
\hline Problema & $\begin{array}{c}\text { Objetivo } \\
\text { específico }\end{array}$ & Resultados & Actividades \\
\hline Alto desempleo & $\begin{array}{l}\text { Bajar nivel de } \\
\text { desempleo en el } \\
\text { Municipio de } \\
\text { Girardota }\end{array}$ & $\begin{array}{l}\text { Mano de obra calificada. } \\
\text { Política pública de empleo. } \\
\text { Nuevas empresas en el } \\
\text { municipio. Competitividad } \\
\text { del sector industrial. Oferta } \\
\text { de empleos en niveles } \\
\text { profesionales } \\
\text { tecnológicos. Ofertas de } \\
\text { empleo para las mujeres. }\end{array}$ & $\begin{array}{l}\text { Formulación Proyectos I+D+i. Programas } \\
\text { de apoyo para el acceso a la educaciór } \\
\text { superior. Creación de programas de apoyo } \\
\text { al emprendimiento. Cursos en formaciór } \\
\text { en competencias laborales. Realización de } \\
\text { convenios con entidades financieras para } \\
\text { el apoyo a la industria y e } \\
\text { emprendimiento. Programa de incentivos } \\
\text { tributarios para empresas que apoyen e } \\
\text { empleo para la mujer. Programas de RSE. }\end{array}$ \\
\hline \multirow[t]{2}{*}{$\begin{array}{l}\text { Baja calidad de la } \\
\text { educación }\end{array}$} & $\begin{array}{lr}\text { Mejorar la } \\
\text { calidad de la } \\
\text { educación en el } \\
\text { Municipio de } \\
\text { Girardota }\end{array}$ & $\begin{array}{l}\text { PEl y PEM articulados con el } \\
\text { sistema productivo. } \\
\text { Compromiso de docentes y } \\
\text { directivos. Inversión en } \\
\text { investigación. Adecuados } \\
\text { sistemas de evaluación. Plan } \\
\text { estratégico Parque. }\end{array}$ & $\begin{array}{l}\text { Formulación de PEM. Actualización de PE } \\
\text { y sistemas de evaluación. Gestión de } \\
\text { recursos para investigación. Programas de } \\
\text { incentivos a docentes. Formulación de } \\
\text { proyectos de investigación con énfasis en } \\
\text { el contexto local. Mejoras en } \\
\text { infraestructura. Convenios con I.E.S. }\end{array}$ \\
\hline & & $\begin{array}{l}\text { Educativo. Aumento calidad } \\
\text { educativa. Creación de una } \\
\text { I.E.S. }\end{array}$ & $\begin{array}{l}\text { Ofrecer programas para el sector rural } \\
\text { Elaboración de plan estratégico para e } \\
\text { Parque Educativo. Mejoramiento de vías } \\
\text { rurales. Convenios con empresas } \\
\text { transportadoras }\end{array}$ \\
\hline $\begin{array}{l}\text { Poca } \\
\text { competitividad } \\
\text { de sectores } \\
\text { claves de la } \\
\text { economía local }\end{array}$ & $\begin{array}{l}\text { Aumentar la } \\
\text { competitividad } \\
\text { de sectores } \\
\text { claves de la } \\
\text { economía en el } \\
\text { municipio de } \\
\text { Girardota }\end{array}$ & $\begin{array}{l}\text { Trabajo colaborativo entre } \\
\text { empresas. Cadenas de } \\
\text { intermediación óptimas. } \\
\text { Aumento de inversión en } \\
\text { l+D+i. Productos y servicios } \\
\text { con valor agregado. } \\
\text { Creación de Clúster Agrícola } \\
\text { e Industrial. Mejoramiento } \\
\text { de vías. Inversión en } \\
\text { sistemas de apoyo a la } \\
\text { producción. Incentivos a la } \\
\text { exportación. }\end{array}$ & $\begin{array}{l}\text { Formulación de política pública para e } \\
\text { fomento de la asociatividad empresarial } \\
\text { Creación de Programas de capacitación } \\
\text { Gestión de recursos para el fomento de I+ } \\
\text { D i i. Programas de apoyo a } \\
\text { mejoramiento continuo. Apoyo para la } \\
\text { creación de Clúster Agrícola e Industrial } \\
\text { Mejoramiento de vías. Construcción de } \\
\text { beneficiaderos, centros de acopio y de } \\
\text { transformación. Creación oficina de } \\
\text { comercio exterior. }\end{array}$ \\
\hline $\begin{array}{l}\text { Poca } \\
\text { participación } \\
\text { ciudadana }\end{array}$ & $\begin{array}{l}\text { Fomentar la } \\
\text { participación } \\
\text { ciudadana en el } \\
\text { Municipio de } \\
\text { Girardota }\end{array}$ & $\begin{array}{l}\text { Mejor imagen de la gestión } \\
\text { pública. Trabajo asociativo } \\
\text { entre comunidades y } \\
\text { gobierno. Solución de } \\
\text { problemas en comunidades. } \\
\text { Bajos niveles de corrupción. } \\
\text { Alta gobernabilidad. } \\
\text { Comunidad participativa. }\end{array}$ & $\begin{array}{l}\text { Formulación de proyectos de impactc } \\
\text { social. Cursos de formación en } \\
\text { participación ciudadana. Programa } \\
\text { Gobierno Transparente. Creación de } \\
\text { asociaciones comunitarias. Estímulos para } \\
\text { la participación ciudadana. Creación de } \\
\text { canales de participación ciudadana } \\
\text { Construcción colectiva de planes de } \\
\text { desarrollo local. }\end{array}$ \\
\hline
\end{tabular}

Fuente: elaboración propia. 
Tabla 5. Matriz DOFA

Table 5. Matrix DOFA

\begin{tabular}{|c|c|c|}
\hline \multicolumn{3}{|c|}{ MATRIZ DOFA } \\
\hline $\begin{array}{l}\text { OBJETIVOS } \\
\text { Bajar niveles de desempleo. } \\
\text { Formular un modelo educativo } \\
\text { local de calidad y pertinente. } \\
\text { Aumentar la competitividad de } \\
\text { sectores claves de la economía. } \\
\text { Fomentar la participación } \\
\text { ciudadana. }\end{array}$ & $\begin{array}{l}\text { FORTALEZAS } \\
\text { Recursos agrícolas. } \\
\text { Sector industrial, Comercial y } \\
\text { Pyme en crecimiento. } \\
\text { Ubicacion geográfica. }\end{array}$ & $\begin{array}{l}\text { DEBILIDADES } \\
\text { Poca competitividad sector agrícola y de } \\
\text { Pyme. Poca asociatividad entre } \\
\text { empresas. Inexistencia de programas de } \\
\text { formación para sectores claves de la } \\
\text { economía local. Ausencia de sistemas de } \\
\text { apoyo a la producción. Poca } \\
\text { participación ciudadana. }\end{array}$ \\
\hline $\begin{array}{l}\text { OPORTUNIDADES } \\
\text { Demanda internacional de } \\
\text { productos agrícolas elaborados. } \\
\text { Llegada de nuevas empresas. } \\
\text { Políticas públicas de apoyo al } \\
\text { Agro y la Industria. Proceso de } \\
\text { paz y postconflicto }\end{array}$ & $\begin{array}{l}\text { POTENCIALIDADES } \\
\text { Tradición panelera. Recursos } \\
\text { agrícolas. Asociaciones agrícolas. } \\
\text { Sector comercio. Presencia de } \\
\text { industria. Pyme en crecimiento. } \\
\text { Llegada empresas. Sector } \\
\text { servicios. Turismo religioso. } \\
\text { Conexión vial. Parque industrial }\end{array}$ & $\begin{array}{l}\text { DESAFIOS } \\
\text { Mejora de la calidad de vida de las } \\
\text { personas. } \\
\text { Aumento de la competitividad local. }\end{array}$ \\
\hline $\begin{array}{l}\text { AMENAZAS } \\
\text { Fuerte competencia externa. } \\
\text { Fluctuación del precio del dólar. } \\
\text { Crisis económicas mundiales. } \\
\text { Desaceleración económica }\end{array}$ & $\begin{array}{l}\text { RIESGOS } \\
\text { Cambios climáticos. } \\
\text { Conflicto armado. } \\
\text { Crecimiento de la población }\end{array}$ & $\begin{array}{l}\text { LIMITACIONES } \\
\text { PEM desarticulado con sistema } \\
\text { productivo. Cultura de subsidiariedad. } \\
\text { Bajos precios agrícolas. Poca preparación } \\
\text { de pequeños empresarios. Poca cultura } \\
\text { de emprendimiento. Informalidad en } \\
\text { Pyme. Acceso a financiamiento para } \\
\text { Pyme. Pocas oportunidades de empleo. } \\
\text { Pocos recursos para la promoción de } \\
\text { programas como «Capital Semilla». }\end{array}$ \\
\hline
\end{tabular}

Fuente: elaboración propia.

\section{Definición de la estrategia de desarrollo local}

Para la formulación de estrategias de desarrollo local, se plantearon líneas de acción generales, como son: formación de la fuerza de trabajo, infraestructuras físicas, gestión de recursos y patrimonio, investigación y tecnología, desarrollo empresarial y calidad de vida. Para tal efecto se construyó una matriz DOFA (Tabla 5), en la cual se resume la información analizada.

Se procedió a construir la matriz de objetivos, estrategias y programas para el desarrollo local (Tabla 6), enumerando aquellas estrategias consideradas más importantes con base en el análisis que se ha hecho, y más adelante, se enuncian los proyectos necesarios para alcanzar los objetivos propuestos.

\section{Elaboración de un plan de inversiones}

Ivan Silva Lira (2003) enumera algunas líneas de proyectos para la elaboración de un plan de inversiones: infraestructuras, empresas locales, recursos humanos locales y desarrollo institucional de la comunidad. Para la identificación de proyectos de inversión se hizo uso de los árboles de medios y fines. La Tabla 7 muestra los objetivos y proyectos propuestos. 
Tabla 6. Matriz de objetivos, estrategias y programas

Table 6. Matrix of objectives, strategies and programs

\begin{tabular}{|c|c|c|}
\hline \multicolumn{3}{|c|}{ MATRIZ DE OBJETIVOS, ESTRATEGIAS YPROGRAMAS PARA EL DESARROLLO LOCAL } \\
\hline \multicolumn{3}{|c|}{ Objetivo general: Mejorar calidad de vida de los habitantes de Girardota } \\
\hline $\begin{array}{l}\text { Objetivos } \\
\text { específicos }\end{array}$ & Estrategias & Programas \\
\hline $\begin{array}{l}\text { Disminuir nivel de } \\
\text { desempleo }\end{array}$ & $\begin{array}{l}\text { Creación de nuevos empleos mediante el } \\
\text { apoyo a Pyme, el fomento del } \\
\text { emprendimiento, la realización de alianzas } \\
\text { estratégicas con empresas y la formación en } \\
\text { competencias laborales. }\end{array}$ & $\begin{array}{l}\text { Reestructuración de la Oficina de Desarrollo } \\
\text { Económico y Centro de Empleo. Fortalecimiento } \\
\text { programa «Capital Semilla». Programas de } \\
\text { acompañamiento y creación de Pyme. Alianzas } \\
\text { para el fomento del empleo y la asociatividad. } \\
\text { Capacitación para buscadores de empleo. }\end{array}$ \\
\hline $\begin{array}{l}\text { Mejorar la calidad } \\
\text { de la educación }\end{array}$ & $\begin{array}{l}\text { Formular un PEM pertinente y articulado con } \\
\text { el sector productivo a través de la } \\
\text { participación de los actores locales. }\end{array}$ & $\begin{array}{l}\text { Planeación estratégica del modelo educativo local. } \\
\text { Fomento de la participación de los actores locales } \\
\text { en la construcción del PEM. Programas de } \\
\text { investigación e innovación. }\end{array}$ \\
\hline
\end{tabular}

Aumentarla Fortalecimiento de sectores claves de la competitividad de sectores claves de la economía local economía local, mediante la implementación de programas de apoyo a la innovación y la generación de valor agregado, así como la mejora creciente de los sistemas locales de apoyo a la producción.
Programa de fomento a la exportación. Planes de incentivos para el fomento de la Innovación empresarial. Plan de mejora de los sistemas de apoyo a la producción local y la formación de clusters locales. Marca Girardota.

\begin{tabular}{ll}
\hline & Establecer canales efectivos de participación \\
Fomentar la & ciudadana para la toma de decisiones y la \\
participación & elaboración de programas para el \\
ciudadana & mejoramiento de la calidad de vida de la \\
& comunidad.
\end{tabular}
Fuente: elaboración propia.
Construcción colectiva de estrategias de desarrollo local. Programas de formación en participación ciudadana. Manejo eficiente del presupuesto participativo. Programa de gobierno transparente. Creación de canales de participación ciudadana.

Tabla 7. Proyectos

Table 7. Projects

OBJETIVO ESPECIFICO

Disminuir nivel de desempleo en el municipio de Girardota.

\section{PROYECTOS}

Formación de mano de obra calificada según necesidades de las empresas. Estudios de necesidades del mercado laboral local. Programa de fomento a creación de Pyme. Capital Semilla. Formulación política pública de empleo. Convenios con el sector financiero para el apoyo a Pyme. Capacitaciones en competencias laborales. Proyectos de incentivos a la innovación. Apoyo a la investigación aplicada. Incentivos a la generación de empleo para la mujer.

Mejorar la calidad de la Actualización PEM. Proyecto de incentivos a docentes. Proyectos de formación en educación en el municipio de Girardota. emprendimiento. Fomento a la investigación aplicada Plan estratégico del Parque Educativo. Proyecto de mejoramiento para los sistemas de evaluación. Inversión en infraestructura de I. E rurales. Creación de una I.E.S.

Aumentar la competitividad de sectores clave de la economía local en el Municipio de Girardota.

Política de fomento de la asociatividad y el trabajo colaborativo entre organizaciones
Grupos de comercialización de productos agrícolas. Proyecto de fomento a I+D + i. Fomento a la creación de Clúster Agrícola e Industrial. Proyecto de gestión tecnológica empresarial. Programa de fomento a la formación de empresas locales. Proyecto de mantenimiento y mejoramiento de vías rurales y de acceso al municipio. Proyecto de incentivos para la actividad exportadora. Marca Girardota.

Fomentar la participación Proyecto de formación en competencias para la participación ciudadana. Talleres de ciudadana en el municipio de Girardota. construcción colectiva de planes de desarrollo local. Programa de transparencia para el manejo del presupuesto participativo. Proyecto de gobierno transparente. Proyecto de mejoramiento y creación de canales de participación ciudadana.

Fuente: elaboración propia. 


\section{CONCLUSIONES}

El procesamiento de la información de los actores señaló como principales problemáticas el mal estado de las vías, la contaminación ambiental, poca participación ciudadana, poca credibilidad en la administración pública, baja calidad de la educación, poca competitividad, microtráfico, la inseguridad y el desempleo; en la zona rural, enfatizaron en las problemáticas relativas al saneamiento básico y el acceso a agua potable. Las soluciones propuestas tienen que ver con la mejora en los esquemas de seguridad y la generación de empleo, la mejora en las vías y movilidad, el aumento de la calidad de la educación, la construcción de alcantarillados y acueductos veredales, la mayor participación ciudadana y el mejoramiento de la competitividad local.

A partir de la identificación de estos problemas se propone estrategias de desarrollo económico local para el municipio de Girardota, se pretende que las mismas constituyan un punto de partida para llevar a cabo la planeación estratégica del territorio con base en sus fortalezas, limitaciones y problemas. Así mismo, la metodología y resultados obtenidos a través de los talleres de construcción de la matriz de importancia y gobernabilidad representan el trabajo directo con los actores locales a través del uso de esta herramienta; además, los proyectos que surgen de los talleres, son un insumo importante a la hora de formular iniciativas para mitigar los problemas priorizados. Otro aspecto a resaltar de las actividades realizadas es el hecho de haber observado el comportamiento de la comunidad en un entorno que facilita su participación para la elaboración de estrategias de desarrollo local acordes con sus necesidades, lo que ayudó a que dichas comunidades empezaran a comprender la importancia de su participación para la toma de decisiones en el municipio de Girardota, Antioquia, Colombia.
Los proyectos que se consideran más relevantes, dado su posible impacto en el desarrollo económico local, así como los instrumentos sugeridos de políticas públicas o herramientas para impulsarlos, son los siguientes:

- Programa de fomento a creación de Pyme, Fomento a la innovación: a partir de políticas como Capital Semilla, asesorías permanentes para acceso a ecosistemas de emprendimiento, alianzas con el sector productivo para el apoyo a Pyme de proveedores y outsourcing, Proyecto de acuerdo para rebaja de cargas impositivas para empresas innovadoras.

- Formación de mano de obra calificada según necesidades de las empresas y estudios de necesidades del mercado laboral local: plan de formación y capacitación permanente, liderado por la Oficina de Empleo Municipal, estudio de caracterización de necesidades del sector productivo local.

- Actualización PEM: este proyecto ya está en proceso, de hecho, se tiene un documento pendiente de aprobación en el Consejo Municipal.

- Plan estratégico del Parque Educativo: dicho plan debe estar fundamentado en los objetivos propuestos cuando se creó el parque educativo, que apuntaban al fomento de la innovación, la investigación, el desarrollo económico y social, y el mejoramiento de la educación en el municipio.

- Inversión en infraestructura de I. E rurales y creación de una I.E.S: en estén sentido ya se están adelantando las obras para la construcción de 2 megacolegios, lo cual supone una mejora importante en el tema de cobertura. En cuanto a la IES local, esto se podría conseguir a partir de la 
reestructuración de la Corporación Politécnica, una institución local que opera en estos momentos en convenio con el SENA, pero que debe apuntar sus esfuerzos de formación en programas pertinentes con la realidad del municipio.

- Política de fomento de la asociatividad y el trabajo colaborativo entre organizaciones y grupos de comercialización de productos agrícolas: si bien ya se tienen conformadas asociaciones de productores agrícolas, estas limitan su funcionamiento a la consecución de ayudas por parte del gobierno local, como utensilios de trabajo, abono, semillas, etc. La formulación de una política de fomento, aprobada mediante acuerdo municipal, debe incluir el enfoque de generación de valor agregado a los productos agrícolas, a partir de procesos de transformación de los mismos, y apuntar a la comercialización no solo a nivel regional y nacional sino internacional.

- Proyecto de fomento a I+D + i: disminución de carga impositiva a empresas con resultados de procesos de $I+D+i$, creación de grupos y semilleros de investigación en las I.E con enfoque en innovación y solución de problemáticas locales, articuladas a su vez con el Parque Educativo Innova.

- Proyecto de mantenimiento y mejoramiento de vías rurales y de acceso al municipio: los recursos para estas obras se pueden gestionar en entidades como el Área Metropolitana y la Gobernación de Antioquia. Se debe hacer énfasis en vías que conecten las zonas veredales con centros de producción y beneficiaderos, así como vías principales.

- Proyecto de incentivos para la actividad exportadora: se debe hacer énfasis en las fortalezas locales, buscando la generación de valor agregado a los productos. De igual manera, se puede motivar la actividad exportadora a partir de beneficios tributarios, y el fomento para la creación de redes de cooperación entre empresas. Un último aspecto es la creación de la Secretaría de Desarrollo Económico, la cual a su vez tenga una dependencia enfocada en el fomento del comercio exterior.

- Proyecto de gobierno transparente: procesos periódicos de rendición de cuentas, consejos comunales, plan de seguimiento a la gestión pública.

- Proyecto de formación en competencias para la participación ciudadana: desarrollado en asocio con las juntas de acción comunal, a través de un plan de formación en mecanismo de participación ciudadana y formulación de planes de desarrollo local de forma conjunta con las comunidades.

El presente estudio puede servir como referente para la construcción de estrategias y la consecución de los objetivos propuestos, a partir de la formulación y puesta en marcha de proyectos como los mencionados anteriormente, así como para la elaboración de otras investigaciones sobre planeación estratégica territorial y desarrollo económico endógeno.

\section{REFERENCIAS}

Alburquerque, F. (2004). El enfoque del desarrollo económico local. Buenos Aires: OIT.

Alburquerque. F. (2004). Desarrollo económico local y descentralización en América Latina. Revista de la CEPAL, 82, p.157171. 
Alburquerque, F.; Dini, M. (2008). Las estrategias de desarrollo económico territorial. Sevilla: Instituto de Desarrollo Regional.

Alcaldía de Girardota. (2016). Plan de Desarrollo Municipal 2016-2019. Girardota: Secretaría de Planeación y Desarrollo Urbano.

Añez, C. (2006). Desarrollo endógeno y empresas transnacionales en la globalización. Aldea Mundo, 11(20), 3748. Recuperado de http://www.redalyc.org/html/543/5430 2005/

Área Metropolitana del Valle de Aburrá. (2016). Plan Estratégico de Empleo para el Valle de Aburra, 2016-2028. Medellín: Servicio Público de Empleo.

Área Metropolitana del Valle de Aburrá. (2017). Observatorio Metropolitano de Información. Recuperado de http://www.metropol.gov.co/observato rio/Paginas/Municipio.aspx

Barroso, M.; Flores, D. (2010). Teoría y Estrategias de Desarrollo Local. Sevilla: Universidad Internacional de Andalucía.

Bigham, R.; Mier, R. (1993) Theories of Local Economic Development: Perspectives From Across the Disciplines. Londres: Sage Publications.

Boissier. S. (1997). El vuelo de una cometa. Una metáfora para una teoría del desarrollo territorial. Revista EURE, XXIII(69), p.729.

Brunet, I. Baltar, F. (2010). Desarrollo endógeno, calidad institucional e innovación, una revisión de la teoría y algunos de sus límites. Revista del CLAD,
(48),115-148. Recuperado de http://www.redalyc.org/html/3575/357 533680005/

Departamento Administrativo Nacional de Estadística. (2013). Informe de Coyuntura Económica Regional Departamento de Antioquia. Medellín: DANE.

Dini, M. (2010). Competitividad, redes de empresas y cooperación empresarial. Revista Gestión Pública, (72), 9-96.

Furio. E. (1994). El desarrollo económico Endógeno y Local: Reflexiones sobre su enfoque interpretativo. Estudios Regionales, (40), 97-112.

Godet, M. (1993). De la anticipación a la acción. Manual de prospectiva y estrategia. Barcelona: Marcombo S.A.

Gorenstein, S.; Burachik, G. (1999). Empleo, pequeñas empresas locales y estrategias de desarrollo endógeno. Revista de Estudios Regionales, (53), 131-157. Recuperado de https://www.researchgate.net/publicati on/227487662_Empleo_pequenas_em presas_locales_y_estrategias_de_desar rollo_endogeno_Experiencias_en_la_Ar gentina

Pérez, M. (2008). La cooperación empresarial en comunidades rurales ¿mecanismo de desarrollo endógeno? Revista Venezolana de Gerencia, 13(41) 9-49. Recuperado de http://www.redalyc.org/html/290/2900 4102/

Quispe, G. Ayaviri, V. (2013). Políticas de desarrollo en los procesos de desarrollo endógeno. Revista Líder, 22, 151-187. Recuperado de 
http://ceder.ulagos.cl/lider/images/nu meros/22/6.-

LIDER\%2022_Quispe_pp151_187.pdf

Romero, I. (2003). Desarrollo endógeno y articulación productiva. Un análisis del sistema productivo andaluz. (Disertación Doctoral). De la base de datos de fondos digitales

(http://fondosdigitales.us.es/media/the sis/513/M_TD-0479.pdf)

Silva, I. (2003). Metodología para la elaboración de estrategias de desarrollo local. Santiago de Chile: Naciones Unidas.

Tello. M. (2006). Las teorías del desarrollo económico local y la teoría y práctica del proceso de descentralización en los países en desarrollo. Recuperado de http://www.pucp.edu.pe/economia/pdf /DDD247.pdf

Vázquez, A. (1998). Política económica local y desarrollo endógeno. Santiago de Chile: CEPAL.
Vázquez. A. (2000). Desarrollo económico local y descentralización. Aproximación a un marco conceptual. Santiago de Chile: CEPAL.

Vázquez. A. (2000). Desarrollo Endógeno y Globalización. Revista EURE, 26(79), p.120.

Vázquez. A. (2007). Desarrollo Endógeno. Teorías y políticas de desarrollo territorial. Investigaciones Regionales, (11) ,183-210.

Vázquez, A. (2009). Desarrollo local, una estrategia para tiempos de crisis. CENES, XXVIII(47), 117-132. Recuperado de http://www.redalyc.org/html/4795/479 549575007/

Vergara, P. (2004). ¿Es posible el desarrollo endógeno en territorios pobres $y$ socialmente desiguales? Ciencias Sociales Online, III(1), 37-52. Recuperado de http://www.uvm.cl./csonline 


\section{Anexo}

Tabla 8. Resumen talleres con la comunidad

Table 8. Summary workshops with the community

\begin{tabular}{|c|c|c|c|}
\hline \multicolumn{4}{|c|}{ Información talleres construcción matriz de Importancia vs Gobernabilidad. } \\
\hline Actividades: & \multicolumn{3}{|c|}{$\begin{array}{l}\text { Formación de grupos de trabajo. } \\
\text { Identificación de problemáticas principales. } \\
\text { Depuración de problemáticas. } \\
\text { Ponderación de Importancia y Gobernabilidad y construcción de matriz IGO. } \\
\text { Presentación de posibles proyectos para solucionar problemáticas. }\end{array}$} \\
\hline Objetivo General: & \multicolumn{3}{|c|}{$\begin{array}{l}\text { Construir la matriz de importancia vs Gobernabilidad como una herramienta para la } \\
\text { identificación de problemáticas del Municipio de Girardota, a partir de información } \\
\text { obtenida de la comunidad. }\end{array}$} \\
\hline Grupo Poblacional & Fecha & Problemáticas principales & Proyectos propuestos \\
\hline $\begin{array}{l}\text { Empleados } \\
\text { Alcaldía Municipio } \\
\text { de Girardota ( } 74 \\
\text { personas, } \\
\text { divididas en } 8 \\
\text { grupos) }\end{array}$ & $\begin{array}{l}16 / 02 \\
/ 2016\end{array}$ & $\begin{array}{l}\text { Baja calidad de la educación, } \\
\text { inseguridad, desempleo, } \\
\text { contaminación ambiental, } \\
\text { poca planeación, poca } \\
\text { participación ciudadana, } \\
\text { falta de cultura ciudadana }\end{array}$ & $\begin{array}{l}\text { Formación sobre participación ciudadana } \\
\text { elaboración del PEM, formación docente } \\
\text { mejoramiento de infraestructura de I.E } \\
\text { aumento cobertura educativa, incentivos a } \\
\text { docentes, control ambiental, fomento a las } \\
\text { Pyme, apoyar procesos de I+D+i, creación de } \\
\text { agencias de desarrollo local, convenios con } \\
\text { empresas, fomento al emprendimiento }\end{array}$ \\
\hline $\begin{array}{lr}\text { Juntas de } & \text { Acción } \\
\text { Comunal }(70 \\
\text { personas }\end{array}$ & $\begin{array}{l}23 / 02 \\
/ 2016\end{array}$ & $\begin{array}{l}\text { Inseguridad, educación, } \\
\text { infraestructura productiva, } \\
\text { desempleo, micro-tráfico, } \\
\text { poca credibilidad en la } \\
\text { administración pública, }\end{array}$ & $\begin{array}{l}\text { mejoramiento de infraestructura de apoyo a } \\
\text { la producción, adecuación de andenes } \\
\text { calles, convenios con el sector productivo } \\
\text { para generación de empleo, programas de } \\
\text { emprendimiento, disminución de impuestos } \\
\text { a empresas, apoyo al sector agrícola } \\
\text { mejoramiento de vivienda }\end{array}$ \\
\hline $\begin{array}{l}\text { Docentes } \\
\text { Directivos } \\
\text { Docentes }\end{array}$ & $\begin{array}{l}24 / 02 \\
/ 2016\end{array}$ & $\begin{array}{l}\text { Calidad educativa, } \\
\text { microtráfico, desempleo, } \\
\text { contaminación, } \\
\text { infraestructura inadecuada } \\
\text { de I.E, movilidad y espacio } \\
\text { público, inequidad social }\end{array}$ & $\begin{array}{l}\text { Actualización del PBOT, adecuación de vías } \\
\text { control público, creación de Pymes, fomento } \\
\text { a la industria, formación en competencias } \\
\text { laborales, reducción de impuestos } \\
\text { empresas, programas de prevención de } \\
\text { consumo de sustancias psicoactivas } \\
\text { formulación del PEM }\end{array}$ \\
\hline $\begin{array}{l}\text { Comunidad en } \\
\text { general ( casi } 200 \\
\text { personas, por lo } \\
\text { tanto el taller se } \\
\text { dividió en dos } \\
\text { sesiones, } \\
\text { realizadas en la } \\
\text { misma fecha) }\end{array}$ & $\begin{array}{l}11 / 03 \\
/ 2016\end{array}$ & $\begin{array}{l}\text { Desempleo, inseguridad, } \\
\text { microtráfico, participación } \\
\text { ciudadana, ralidad } \\
\text { educación, ordenamiento } \\
\text { territorial, atención en salud, } \\
\text { movilidad, vivienda, } \\
\text { credibilidad sector público, } \\
\text { contaminación }\end{array}$ & $\begin{array}{l}\text { Capacitación docente, estímulos para e } \\
\text { estudio, mejoramiento de infraestructura } \\
\text { educativa, capacitación en competencias } \\
\text { laborales, fomento al empleo, proyectos de } \\
\text { vivienda de interés social, mejoramiento y } \\
\text { construcción de vías, programas de } \\
\text { emprendimiento, control ambiental, gestión } \\
\text { de recursos para mejoramiento de la calidac } \\
\text { de vida }\end{array}$ \\
\hline
\end{tabular}

Fuente: elaboración propia. 